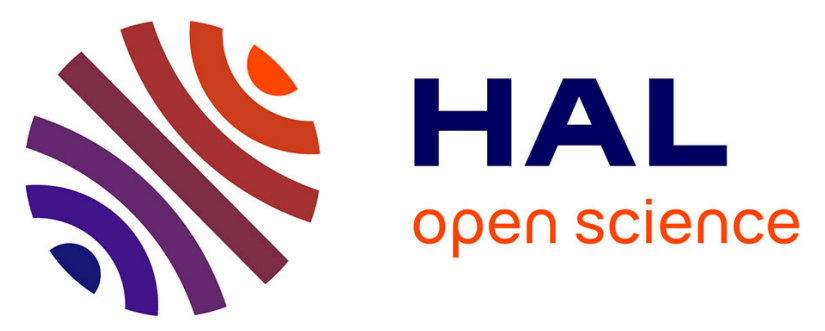

\title{
Production of Scandium-44m and Scandium-44g with Deuterons on Calcium-44: Cross Section measurements and Production Yield Calculations
}

\author{
C. Duchemin, Arnaud Guertin, Ferid Haddad, Nathalie Michel, Vincent \\ Métivier
}

\section{To cite this version:}

C. Duchemin, Arnaud Guertin, Ferid Haddad, Nathalie Michel, Vincent Métivier. Production of Scandium-44m and Scandium-44g with Deuterons on Calcium-44: Cross Section measurements and Production Yield Calculations. Physics in Medicine and Biology, 2015, 60, pp.6847-6864. 10.1088/0031-9155/60/17/6847 . in2p3-01188671

\section{HAL Id: in2p3-01188671 \\ https://hal.in2p3.fr/in2p3-01188671}

Submitted on 26 Jan 2021

HAL is a multi-disciplinary open access archive for the deposit and dissemination of scientific research documents, whether they are published or not. The documents may come from teaching and research institutions in France or abroad, or from public or private research centers.
L'archive ouverte pluridisciplinaire HAL, est destinée au dépôt et à la diffusion de documents scientifiques de niveau recherche, publiés ou non, émanant des établissements d'enseignement et de recherche français ou étrangers, des laboratoires publics ou privés. 


\title{
Production of Scandium-44m and Scandium-44g with Deuterons on Calcium-44: Cross Section Measurements and Production Yield Calculations
}

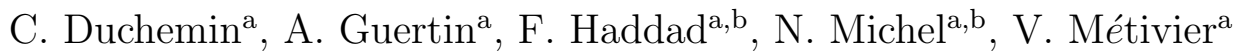 \\ ${ }^{a}$ SUBATECH, Ecole des Mines de Nantes, Université de Nantes, CNRS/IN2P3, Nantes, \\ France \\ ${ }^{b}$ GIP Arronax, 1 rue Aronnax, 44817 Saint-Herblain, France
}

\begin{abstract}
HIGHLIGHTS

- Production of Sc-44m, Sc-44g and contaminants.

- Experimental values determined using the stacked-foil technique.

- Thick Target production Yield (TTY) calculations.

- Comparison with the TALYS code version 1.6.
\end{abstract}

\section{ABSTRACT}

Among the large number of radionuclides of medical interest, Sc-44 is promising for PET imaging. Either the ground state Sc-44g or the metastable state Sc- $44 \mathrm{~m}$ can be used for such application, depending on the molecule used as vector. This study compares the production rate of both Sc-44 states, when protons or deuterons are used as projectiles on an enriched Calcium-44 target. This work presents the first set of data for the deuteron route. The results are compared with the TALYS code. The Thick Target production Yields of Sc-44m and Sc-44g are calculated and compared with the proton route for three different scenarios: the production of Sc-44g for conventional PET imaging, its production for the new $3 \gamma$ imaging technique developed at the SUBATECH laboratory and the production of the Sc- $44 \mathrm{~m} / \mathrm{Sc}-44 \mathrm{~g}$ in-vivo generator for antibody labelling.

Email address: Charlotte.Duchemin@subatech.in2p3.fr (C. Duchemin) 
Keywords: Ca-44 target, Sc-44m/Sc-44g in-vivo generator, deuteron beam, stacked-foil technique, excitation function, TALYS 1.6

\section{Introduction}

Nuclear medicine is a specialty involving the use of radionuclides for diagnosis as well as for therapy of cancers. In the last years, the Positron Emission Tomography (PET) diagnosis technique has been developed rapidly through the use of fluorine-18. At the same time, molecular therapy using new isotopes like Lu-177 has shown very promising results. In this case, the radionuclide must have a half-life which matches that of the vector distribution (peptide or antibody). The combination of imaging information and therapeutic use of radionuclides is called theranostic and allows to personalize the treatment to each patient. Indeed, the diagnosis test, done prior to the treatment, permits to follow and control the patient response to the injected radiopharmaceutical. It allows a better control of the targeting and increases the benefit/toxicity ratio as useless treatments on patients with no response to the diagnosis test are avoided. For a better description of the tracer biodistribution, pairs of radionuclides of the same element are preferable.

Scandium is an useful element for this theranostic concept. Scandium47 can be used for therapy while Sc- 43 and Sc-44 can be used for Positron Emission Tomography (PET). Scandium-47 is a $\beta$ - emitter $(100 \%)$ with a half-life of $\mathrm{T}_{1 / 2}=3.3492$ days which decays to Ti-47. Its $\beta$ energy makes it well suited for radionuclide therapy. Associated to its decay, a $159 \mathrm{keV}$ $\gamma$-ray is emitted (branching ratio of $68 \%$ ) which can be used for imaging in Single Photon Emission Computed Tomography (SPECT). This isotope can be produced efficiently in nuclear reactor (Muller C. et al, 2014).

Scandium-43, with a half-life of 3.9 hours, is a $\beta+$ emitter with a branching ratio of $88.1 \%$. Its production is quite difficult using accelerator since it needs expensive Ca-43 enriched target (Majkowska-Pilip and Bilewicz, 2011). Scandium-44 ground state, scandium-44g or Sc-44g, with a half-life of 3.97 hours, is also a $\beta+$ emitter (branching ratio of $94 \%$ ). This radioisotope can be produced via a generator system Ti-44/Sc-44g (Roesch et al., 2012) but also directly via the irradiation of an enriched Calcium-44 target (Muller C. et al, 2014, Alliot et al., 2015). Scandium-44g has an important production advantage over scandium- 43 because the starting material, Ca-44, is more 
abundant $(2.1 \%)$ than Ca-43 $(0.14 \%)$ leading to less expensive target material. Sc-44g is complementary to other PET isotopes. Indeed, the Sc-44g half-life is between that of Ga-68 $\left(\mathrm{T}_{1 / 2}=67.71 \mathrm{~min}\right)$ and $\mathrm{Cu}-64\left(\mathrm{~T}_{1 / 2}=\right.$ $12.701 \mathrm{~h})$.

Associated to Sc-44g decay, a high energy gamma ray at $1.157 \mathrm{MeV}$, with a high probability of occurrence $(99.9 \%)$, is emitted. This $\gamma$ ray can be used in addition to the two $511 \mathrm{keV}$ resulting from the positron annihilation, on the new three-gamma imaging technique developed at the SUBATECH laboratory (Nantes, France). The aim of this technique is to measure, on an event by event basis, the emitter location in three dimensions as proposed by Grignon C. et al., 2007. To do that, it is necessary to combine the line of response obtained with the two gammas following the positron/electron annihilation with the arrival direction of the third gamma emitted by the radio-isotope. A first device using liquid xenon as detection medium has been developed to show the feasibility of this new technique, called XEMIS for XEnon Medical Imaging System. Simulations have shown the possibility to reduce the injected dose to the patient with a good energy and spatial resolution (Oger et al., 2012). Today, an experimental device is in construction for the small animal imaging, named XEMIS 2 (Gallego Manzano et al., 2014).

Last but not least, with scandium-44, it is possible to match the halflife of the isotope to the vector characteristics. The 3.97 hours half-life of Sc- $44 \mathrm{~g}$ is well suitable for the use of peptide and small molecule with rapid distribution on the body. For long biological processes like the ones induced when antibodies are used, its metastable state, Sc- $44 \mathrm{~m}$, can be used as an in-vivo generator of Sc-44g. This excited state of scandium has a half-life of 58.6 hours and decays mainly by Internal Transition (IT) to its ground state Sc-44g. It has been shown that when a molecule is labeled with Sc-44m, the Sc-44g formed by its decay remains attached to this molecule (HuclierMarkai et al., 2014). Sc-44m cannot be formed through the Ti-44/Sc-44 generator. It can be produced, at the same time as Sc-44g, with particle accelerators interacting on $\mathrm{Ca}-44$ enriched target. Experimental data exist on the production of scandium-44 using protons as projectiles on Ca-44 (Mitchell et al., 1982, Levkovskij et al.1, 1991 and Krajewski et al., 2013). They are in good agreement with the assessment given by the TALYS code version 1.6 (Koning and Rochman, 2012). From these cross section values, the thick target production yields (TTY) (See Section 2.3) have been calculated to be $23 \mathrm{MBq} / \mu \mathrm{A} . \mathrm{h}$ for Sc-44m and $2.7 \mathrm{GBq} / \mu \mathrm{A}$.h for Sc-44g at $30 \mathrm{MeV}$ (See 
figure 1).

In some cases, deuterons can be advantageously used for isotope production (Alliot et al., 2015). This is the case for Copper-64 production from Nickel-64 enriched target as well as for the production of Re-186g using a Tungsten-186 enriched target. In this latter case, 3.7 times more activity can be obtained with deuterons of $17.6 \mathrm{MeV}$ than with protons at the same energy (Duchemin et al., 2015). In order to investigate the potential advantage in using deuterons and due to the lack of experimental data with deuterons as projectiles, we have used the TALYS code to get hints on the best production route between proton and deuteron. The TALYS results for the Ca-44 $(\mathrm{d}, 2 \mathrm{n})$ reactions are plotted in figure 2 . The TTY calculated from these theoretical values is $65 \mathrm{MBq} / \mu \mathrm{A}$.h for $\mathrm{Sc}-44 \mathrm{~m}$ and $2.3 \mathrm{GBq} / \mu \mathrm{A}$.h for Sc- $44 \mathrm{~g}$ at $30 \mathrm{MeV}$, showing a ratio Sc-44m/Sc-44g 3.3 times higher using deuterons than protons for this energy.

The aim of this work is to experimentally determine the Sc- $44 \mathrm{~g}$ and Sc$44 \mathrm{~m}$ production cross sections using deuterons on an enriched Ca-44 target in order to be able to calculate the associated production yields, and make a comparison with the proton route. Experimental data have been extracted up to $34 \mathrm{MeV}$ for the Ca-44(d,2n)Sc-44m,Sc-44g reactions, using the stackedfoil technique and gamma-spectrometry, at the ARRONAX cyclotron. Some results on the production of K-42,43 and Sc- 43 have been also obtained but not in all the targets as the main objective was to properly measure the activity of Sc-44m and Sc-44g. Based on these new data, three different scenarios for the production of Sc-44 have been defined depending on the aim of its use: the first one corresponds to the use of Sc-44g for PET imaging with small molecules as vectors. In this case, the production of the metastable state $\mathrm{Sc}-44 \mathrm{~m}$ has to be limited. The second one corresponds to the use of Sc- $44 \mathrm{~g}$ for the new three-gamma imaging technique, developed at the SUBATECH laboratory (Nantes, France). In this case, Sc-43 and Sc-44m will generate background in the detection system as they don't have the third $\gamma$ emission with the energy required. The third one corresponds to the use of the Sc$44 \mathrm{~m} / \mathrm{Sc}-44 \mathrm{~g}$ in-vivo generator to follow long biological processes. In this case, it is necessary to maximize the Sc-44m production. All our results are compared with the results of the TALYS code version 1.6, as these information were used to infer the potential interest of deuterons over protons. 

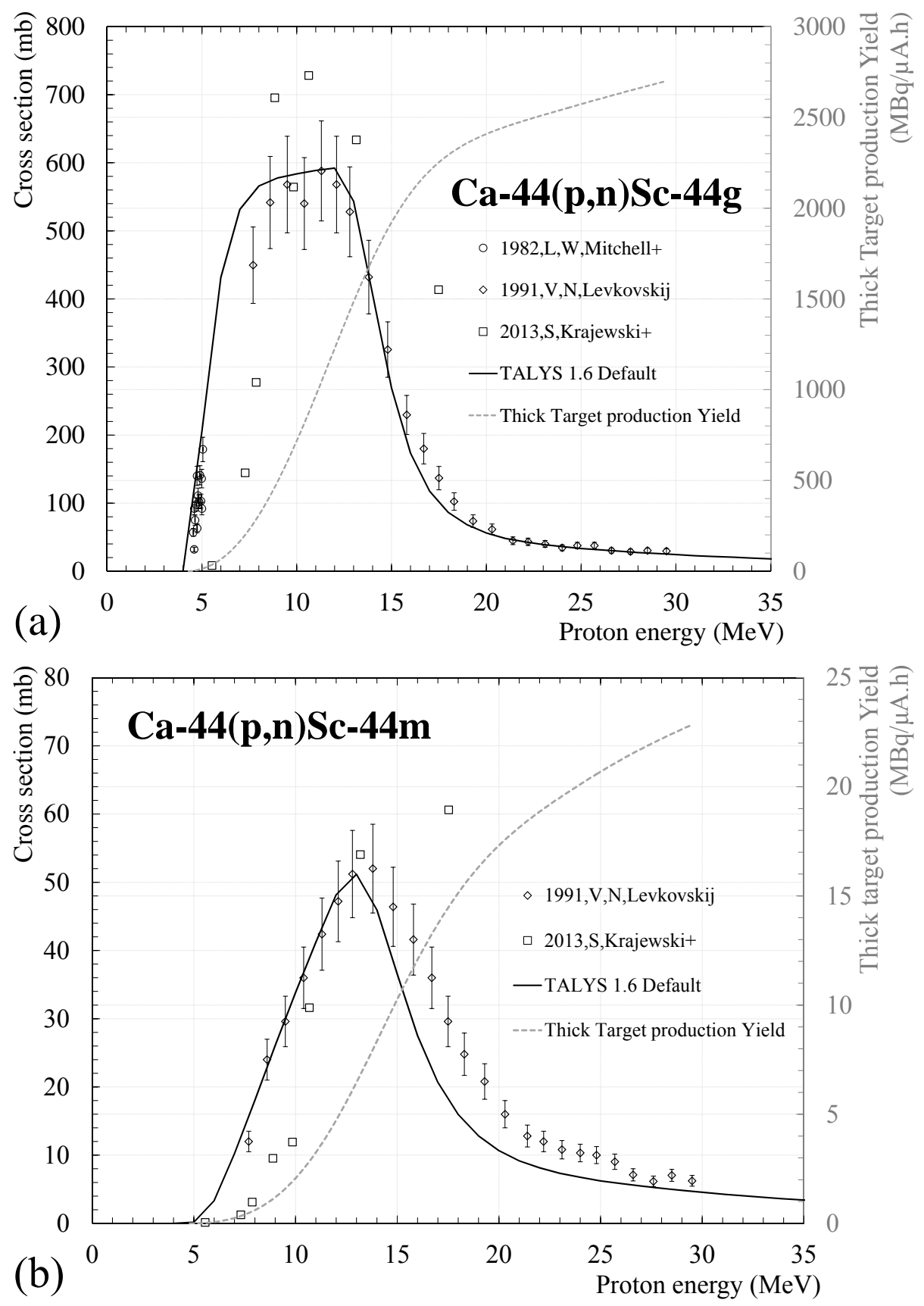

Figure 1: Experimental excitation functions for the Ca-44(p,n)Sc-44g (a) and Ca$44(\mathrm{p}, \mathrm{n}) \mathrm{Sc}-44 \mathrm{~m}$ (b) reactions with their calculated thick target production yield, compared with TALYS 1.6 with default models 


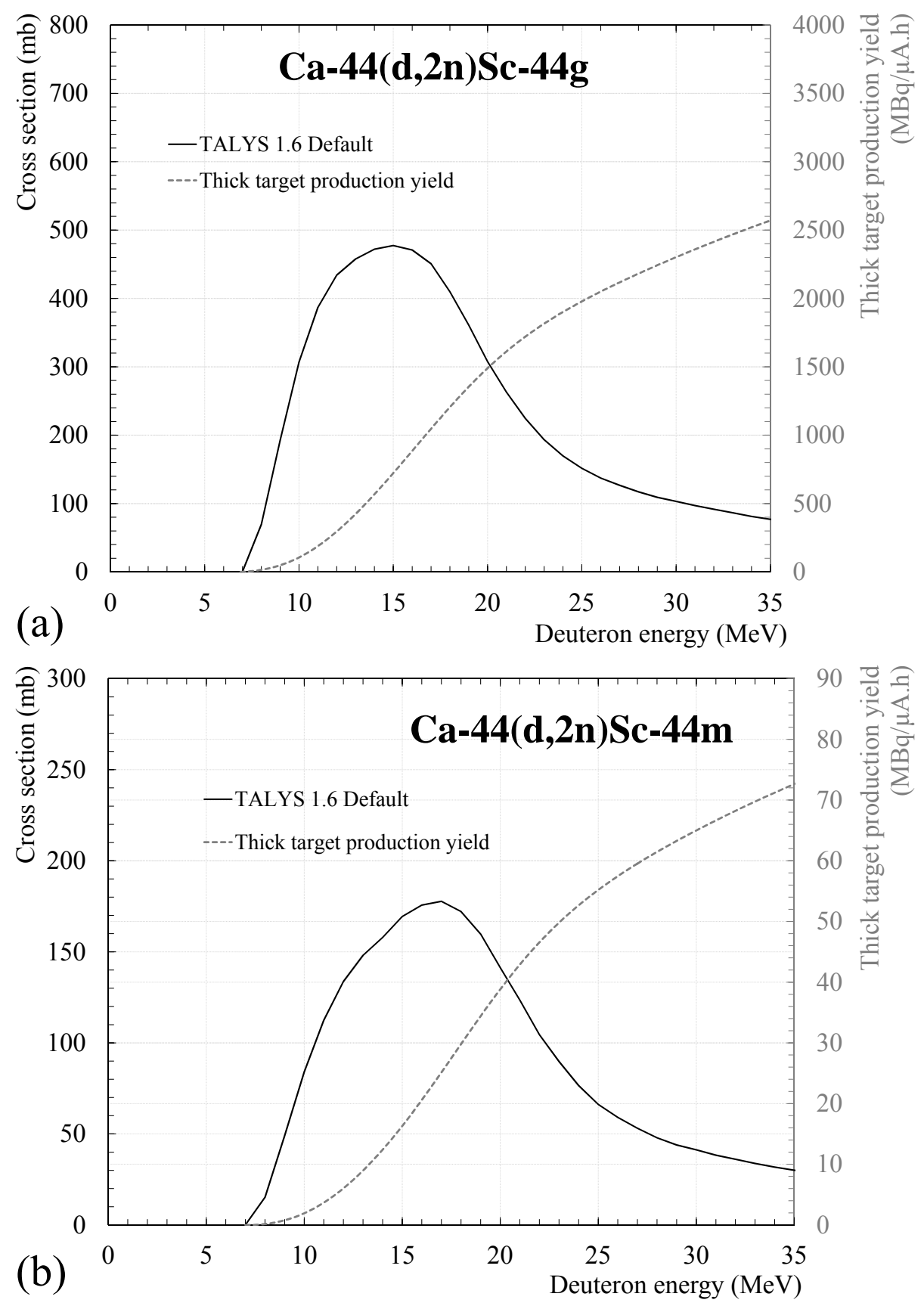

Figure 2: Estimations of the excitation functions of the Ca-44(d,2n)Sc-44g (a) and Ca$44(\mathrm{~d}, 2 \mathrm{n}) \mathrm{Sc}-44 \mathrm{~m}$ (b) reactions from TALYS 1.6 with default parameters and the associated thick target production yield 


\section{Materials and methods}

\subsection{Experimental set-up}

In this work, Sc-44g and Sc-44m are produced using an enriched Ca-44 carbonate target $\left(\mathrm{CaCO}_{3}\right)$ from Chemgas ${ }^{\circledR}$. The isotopic composition of the calcium powder is reported in Table 1.

Table 1: Isotopic composition of the calcium carbonate powder from Chemgas ${ }^{\circledR}$

\begin{tabular}{ccccccc}
\hline Isotope & Ca-40 & Ca-42 & Ca-43 & Ca-44 & Ca-46 & Ca-48 \\
$\%$ & 0.92 & 0.02 & 0.01 & 99.04 & $\leq 0.01$ & 0.01 \\
\hline
\end{tabular}

$99.04 \%$ of the calcium target is composed by Ca-44. Ca-40 represents the vast majority of the other $\mathrm{Ca}$ isotopes present in the target with $0.92 \%$. All isotopes investigated in this work are not produced from $\mathrm{Ca}-40$. The parasitic reactions are on $\mathrm{Ca}-43$ and $\mathrm{Ca}-42$. Sc-44 is produced via $\mathrm{Ca}-44(\mathrm{~d}, 2 \mathrm{n})$ and $\mathrm{Ca}-$ $43(\mathrm{~d}, \mathrm{n})$ reactions. The contribution on this latter isotope is negligible since Ca-43 is present at a level of only $0.01 \%$. The same holds for the production of Sc-43 which can be made via Ca-44(d,3n), Ca-43(d,2n) or Ca-42(d,n) $(0.02 \%)$. Both Ca-43 and Ca-42 are present at a low level in the target and their contribution can be neglected. Same comments can be made for the production of K-42 and K-43. The calcium carbonate targets have been prepared at the Institut de Physique Nucléaire dOrsay (IPNO, France) by the CACAO (Chimie des Actinides et Cibles radioActives à Orsay) project team (Petitbon-Thévenet, 2010). The backing of our target was chosen to be 100 micrometers thick aluminum foils with a surface of $25^{*} 25 \mathrm{~mm}^{2}$, manufactured

by the Goodfellow Company ${ }^{\circledR}$. The backing surface was first prepared to be sufficiently rough to insure a good adhesion of the carbonate calcium deposit. Before use, each support was cleaned with isopropanol. To perform the centrifugation/sedimentation, the apparatus of the Centre de Spectrométrie Nucléaire et de Spectrométrie de Masse in Orsay (France) was used. The experimental process is exposed in the article of Durnez, Petitbon-Thévenet and Fortuna published in 2014. An amount of $6.53 \mathrm{mg}$ of calcium carbonate powder was used to make each target. This value corresponds to $5.44 \mathrm{mg}$ with $20 \%$ of losses during the process. One milliliter of a mix of both chloroform and polystyrene $(17 \mathrm{~g} / \mathrm{l})$ as well as two milliliters of ethyl acetate were added to the powder. This mix is transferred to a bottle and placed in an ultrasonic bath for one hour to produce the good quality slurry. Then the solution was transferred to the centrifuge tube mounted with the backing. The tube with 
slurry was placed in the centrifuge and was spun at 3500 revolutions per minute during one hour. After completing the sedimentation, the excess of the solvent is removed and the target is left aside for polymerizing during twelve hours. At the end of the process, the amount of target material is defined by precise weighting. Typical values are 2.54 (12) $\mathrm{mg} . \mathrm{cm}^{-2}$ for our 10 targets.

At the ARRONAX cyclotron (Haddad et al., 2008), the cross section measurements are made using the stacked-foil method (Duchemin et al., 2015, Blessing et al., 1995) which consists in the irradiation of a set of thin foils, grouped as patterns. Each pattern contains a target to produce the isotopes of interest, a thin aluminium cover to protect the calcium carbonate deposit, a monitor foil to have information on the beam current through the use of a reference reaction recommended by the International Atomic Energy Agency and a degrador to change the incident beam energy from one target foil to the next one. Each monitor, deposit protection and degrador foil has been weighed before irradiation using an accurate scale $\left( \pm 10^{-5} \mathrm{~g}\right)$ and scanned to precisely determine its area. The thickness is deduced from these values, assuming it is homogeneous over the whole surface. In this work, $10 \mu \mathrm{m}$ thick titanium monitor foils and 100 to $500 \mu \mathrm{m}$ thick aluminium degrador foils were irradiated. The titanium and aluminium foils, with a purity of 99.6 $\%$ and $99 \%$, respectively, were purchased from Goodfellow ${ }^{\circledR}$.

The ARRONAX cyclotron delivers deuteron beams with an energy uncertainty of $\pm 0.25 \mathrm{MeV}$, as specified by the cyclotron provider using simulations. The beam line is under vacuum and closed using a $75 \mu \mathrm{m}$ thick kapton foil. The stacks were located about $7 \mathrm{~cm}$ downstream in air. The energy through each foil has been determined in the middle of the thickness of the foil using the SRIM software (Ziegler et al., 2010). Energy loss in the kapton foil and air have been taken into account. All along the stack, depending on the number of foils, the energy uncertainty calculated using the SRIM software (Ziegler et al., 2010) increases up to $\pm 0.61 \mathrm{MeV}$ due to the energy straggling (see Table 4). Three stacks were irradiated with a different incident energy in order to minimize this energy dispersion and cover the energy range from $34 \mathrm{MeV}$ down to $8 \mathrm{MeV}$, which corresponds to the whole energy range of interest (see Table 2). Irradiations were carried out with a mean beam intensity between 54 to 68 nA during 30 minutes. The beam current through the target has been limited knowing that $\mathrm{CaCO}_{3}$ can be affected by a thermal decomposition above 800 Celsius degrees. In the worst case, the maximal power to the target was $7 \mathrm{~mW} / \mathrm{cm}^{2}$ which is 
not enough to decompose the calcium carbonate. Irradiation conditions are reported in Table 2 .

Table 2: Irradiation conditions and energies estimated with SRIM

\begin{tabular}{cc|cccc}
\hline $\begin{array}{c}\text { Beam energy } \\
(\mathrm{MeV})\end{array}$ & $\begin{array}{c}<\text { Intensity } \\
(\mathrm{nA})\end{array}$ & \multicolumn{4}{|c}{$\begin{array}{c}\text { Energy points } \\
(\mathrm{MeV})\end{array}$} \\
\hline $34.00(25)$ & 54 & $33.33(28)$ & $29.45(44)$ & $25.11(54)$ & $21.66(60)$ \\
$20.50(25)$ & 55 & $19.51(29)$ & $17.33(36)$ & $14.91(44)$ & $12.17(58)$ \\
$16.90(25)$ & 68 & $10.71(49)$ & $8.40(61)$ & & \\
\hline
\end{tabular}

For all the experiments, the recommended cross section of the reaction Ti-nat(d,x)V-48 (Tárkányi et al., 2001) is used to obtain information on the beam current from the $\mathrm{Ti}$ monitor foils. This reaction is well known over the whole studied energy range. A Faraday cup was placed after the stack to collect charges and control the intensity during the irradiation. The cross section values obtained with this measurement are in agreement with those obtained using the monitor foil within $6.5 \%$ in average. This difference is explained by the beam stop used to collect the beam current after the stack which has no electron suppression device. In the stacks, $20 \mu \mathrm{m}$ aluminium foils were placed in front of the Ca-44 carbonate deposit, for protection. The activity on Ca-44 were determined measuring the target and the protection cover together. The activity measurements in each foils were performed using a high purity germanium detector from Canberra (France) with lowbackground lead and copper shielding. All foils were counted twice. The first measurements started the day after the irradiation during one hour, for all target, protection cover and monitor foils. The second ones were performed for a minimum of 24 hours (one day) and up to 60 hours (during the week-end), one week after the end of irradiation for the Ca-44 targets and after three weeks for the monitor foils, waiting for the complete decay of Scandium-48. Indeed, Sc-48 ( $\mathrm{T}_{1 / 2}=43.67$ (9) hours) has common gamma lines with the radioisotope of reference, Vanadium-48 $\left(\mathrm{T}_{1 / 2}=15.9735(25)\right.$ days). Gamma spectra were recorded in a suitable geometry calibrated in energy and efficiency with standard Co-57,60 and Eu-152 gamma sources from LEA-CERCA (France). The full widths at half maxima were $1.04 \mathrm{keV}$ at $122 \mathrm{keV}$ (Co-57 $\gamma$ ray) and $1.97 \mathrm{keV}$ at $1332 \mathrm{keV}$ (Co-60 $\gamma$ ray). Samples were placed at a height of $19 \mathrm{~cm}$ from the detector in order to reduce the dead time and the effect of sum peaks. The dead time during the counting was always kept below $10 \%$. 


\subsection{Data processing}

The activity values of the produced radioisotopes were derived from the $\gamma$ spectra and the nuclear decay data (National Nuclear Data Center; Ekström and Firestone, 2004) given in Table 3 using the Fitzpeak spectroscopy software (FitzPeaks Gamma Analysis and Calibration Software version 3.66). Knowing the precise thickness of the foil and the activity value of the produced isotope, its production cross section is calculated using the activation formula (1) with the appropriate beam current:

$$
\sigma=\chi \cdot \frac{A c t \cdot A}{\Phi \cdot N_{a} \cdot M \cdot\left(1-e^{-\lambda t}\right)}
$$

In this equation, the production cross section $\sigma$ of a radioisotope depends on its measured activity reported to the time at the end of irradiation (Act), its decay constant $(\lambda)$, its atomic mass $(A)$, its areal density $(\mathrm{M})$, its chemical and isotopic purity $(\chi)$, the irradiation duration $(\mathrm{t})$ and the beam current $(\Phi)$.

In our experiment, each target foil received the same beam current as the monitor foil that follows. It is then easier to use the relative equation (2) in which the knowledge of the beam current is no longer necessary. In this equation, the prime parameters are associated to V-48 while the others relate to the radioisotope produced in the target (i.e. Sc-44m, Sc-44g, Sc-43, $\mathrm{K}-42$ and $\mathrm{K}-43)$.

$$
\sigma=\sigma^{\prime} \cdot \frac{\chi^{\prime} \cdot A c t \cdot A \cdot M^{\prime} \cdot\left(1-e^{-\lambda^{\prime} t}\right)}{\chi \cdot A c t^{\prime} \cdot A^{\prime} \cdot M \cdot\left(1-e^{-\lambda t}\right)}
$$

The cross section uncertainty is estimated with a propagation error calculation. Since all the parameters of equation (2) are independent, the total error is expressed as a quadratic sum (Eq.(3)). The main error sources come from the recommended cross section, the activity values of each produced radioisotopes and the foils thickness uncertainties. An uncertainty around $11 \%$ is applied to the recommended cross section values given by the uncertainty of the nearest experimental value. The uncertainties on the activity of the radio-isotopes produced on the Ca-44 targets depend on different parameters as the gamma line branching ratio and energy, half-life etc, described afterwards. These uncertainties are, in average, of $3.5 \%$ for Sc-44m activity, $16 \%$ for Sc-44g, $20 \%$ for K-42, $18 \%$ for K-43 activity and up to $30 \%$ for Sc-43. The uncertainty on the activity of V-48 is $4 \%$ in average. Around 1 
$\%$ of uncertainty is calculated for the areal density. The contribution of the irradiation time uncertainty is not significant and has been neglected.

$$
\frac{\Delta \sigma}{\sigma}=\sqrt{\left(\frac{\Delta \sigma^{\prime}}{\sigma^{\prime}}\right)^{2}+\left(\frac{\Delta A c t}{A c t}\right)^{2}+\left(\frac{\Delta A c t^{\prime}}{A c t^{\prime}}\right)^{2}+\left(\frac{\Delta M}{M}\right)^{2}+\left(\frac{\Delta M^{\prime}}{M^{\prime}}\right)^{2}}
$$

\subsection{Thick Target production Yield (TTY)}

Using the cross section values obtained in this work or those in databases as a function of the energy $\sigma(\mathrm{E})$, we have calculated the associated Thick Target production Yields in $\mathrm{Bq} / \mu \mathrm{A}$.h, also called production yield or integral yield. The values are obtained as a function of the projectile energy, using the relation (4).

$$
T T Y=\Phi \cdot \chi \cdot \frac{N_{a} \cdot \rho}{A}\left(1-e^{-\lambda \cdot t}\right) \int_{E \min }^{E \max } \frac{\sigma(E)}{\frac{d E}{d x}} d E
$$

In relation (4), $\phi$ is set to be the number of particles per second delivered in one $\mu \mathrm{A} . \chi$ corresponds to the isotopic and chemical purity of the target and $\mathrm{N}_{a}$ is the Avogadro number. The TTY results presented in this article are obtained using the density $\rho$ and the atomic mass A of a calcium- 44 carbonate target. The density is provided by the CACAO team who prepared the target and fixed at $2.71 \mathrm{~g} . \mathrm{cm}^{-3}$. All the TTY calculation is done taking into account one hour of irradiation, which is less that the time where the activity of the investigated radionuclides is saturated. $\frac{d E}{d x}$ is the specific energy loss of the projectile in the target material $\left(\mathrm{MeV} . \mathrm{cm}^{-1}\right)$. In a thick target, the incident particle energy decreases with the penetration depth. $\mathrm{E}_{\max }$ corresponds to the incident projectile energy when it enters into the target whereas $\mathrm{E}_{\text {min }}$ corresponds to its energy when it leaves the target. TTY is also called production yield or integral yield.

\subsection{Comparison with the TALYS 1.6 code}

In this work, all the experimental cross section values are compared with the latest version (1.6) of the TALYS code released in December, 2013 (Koning and Rochman, 2012). TALYS is a nuclear reaction program to simulate reaction induced by light particles on nuclei heavier than carbon. It incorporates theoretical models to predict observables including theoretical cross 
section values as a function of the incident particle energy (from $1 \mathrm{keV}$ to 1 $\mathrm{GeV}$ ). A combination of models that better describes the whole set of data available for all projectiles, targets and incident energies have been defined by the authors of the code and put as default in the code. This way, the code can be launched with minimum information in the input file: the projectile type and its incident energy, the target type and its mass. The experimental data obtained in this work are compared to TALYS with default models (named TALYS 1.6 Default in the graphs).

\section{Results and discussions}

Production cross sections are needed to obtain the Thick Target production Yield for a produced radio-isotope. In a first part, new results on deuteron induced Sc-44 production cross section on Ca-44 will be exposed, as well as the contaminant cross sections. In a second part, the Sc-44m and Sc-44g TTY will be calculated and compared with proton routes for different production scenarios.

\subsection{Production cross sections for deuteron induced reactions}

The production cross sections of the produced radioisotopes are plotted in figures 3 to 7 as full circles and the associated numerical values are reported in Table 4.

\subsubsection{Production cross section of Sc-44m}

Sc-44m $\left(\mathrm{T}_{1 / 2}=58.6(1) \mathrm{h}\right)$ mainly decays by Internal Transition (IT), at $98.80(7) \%$, to its ground state Sc-44g $\left(\mathrm{T}_{1 / 2}=3.97(4) \mathrm{h}\right)$ by emitting an intense $\gamma$ ray at $271.13 \mathrm{keV}$ (Table 3). It also decays by Electron Capture (EC) process $(1.20(7) \%$ ) to Ca-44 (stable) by emitting three $\gamma$ rays of 1001.83 (1.20\%), 1126.06 (1.20\%) and $1157.031 \mathrm{keV}(1.20 \%)$. The high branching ratio of the $271.13 \mathrm{keV}$ gamma line allows to use it to extract the

Sc-44m production cross section plotted in figure 3, with the results of the TALYS code version 1.6 with default parameters.

The Sc-44m production cross section shows a maximum at $17.3 \mathrm{MeV}$ with a value of $185 \mathrm{mb}$ (see figure 3 ). The TALYS code gives cross section values slightly shifted to lower energies as compared to those experimentally determined (see figure 2(a)). The magnitude of the cross section is of the same order for both experimental and TALYS results. 


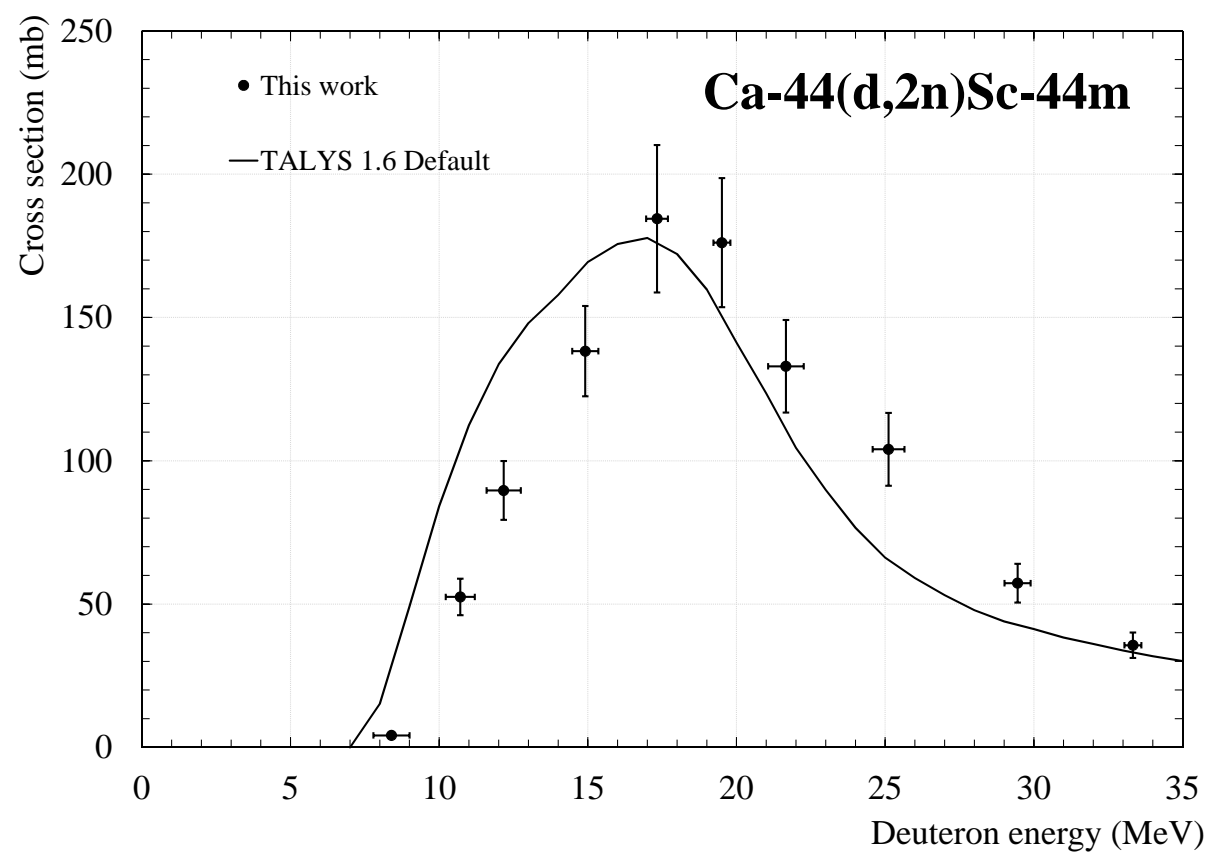

Figure 3: Excitation function for the Ca-44(d,2n)Sc-44m reaction

\subsubsection{Direct production cross section of $S c-44 \mathrm{~g}$}

Sc-44g $\left(\mathrm{T}_{1 / 2}=3.97(4) \mathrm{h}\right)$ is a $100 \% \mathrm{EC} / \beta+$ emitter which decays to $\mathrm{Ca}-44$ (stable). It emits an intense gamma ray at $1157.031 \mathrm{keV}(99.9$ $\%$ ), used to determine its activity. The measured activity and the cross section obtained in this work correspond to the direct production of Sc- $44 \mathrm{~g}$ in a Ca- 44 carbonate target. As Sc- $44 m$ decays to Sc- $44 \mathrm{~g}$, its contribution has been determined and removed from the Sc-44g activity, as well as the contamination by the direct decay of Sc- $44 \mathrm{~m}$ to Ca-44 in the gamma line at $1157 \mathrm{keV}$, which is common to $\mathrm{Sc}-44 \mathrm{~m}\left(\mathrm{I}_{\gamma}=1.2 \%\right)$ and $\mathrm{Sc}-44 \mathrm{~g}$. The experimental Sc-44g production cross section is plotted in figure 4.

In figure 4, the experimental data show a peak broader with a maximum $10 \%$ higher than the results obtained using the TALYS calculations. The maximum of the experimental cross section is found to be $538 \mathrm{mb}$ at 14.9 $\mathrm{MeV}$, against $470 \mathrm{mb}$ with TALYS for the same incident deuteron energy. In this case, there is no energy shift between TALYS and the experimental results. 


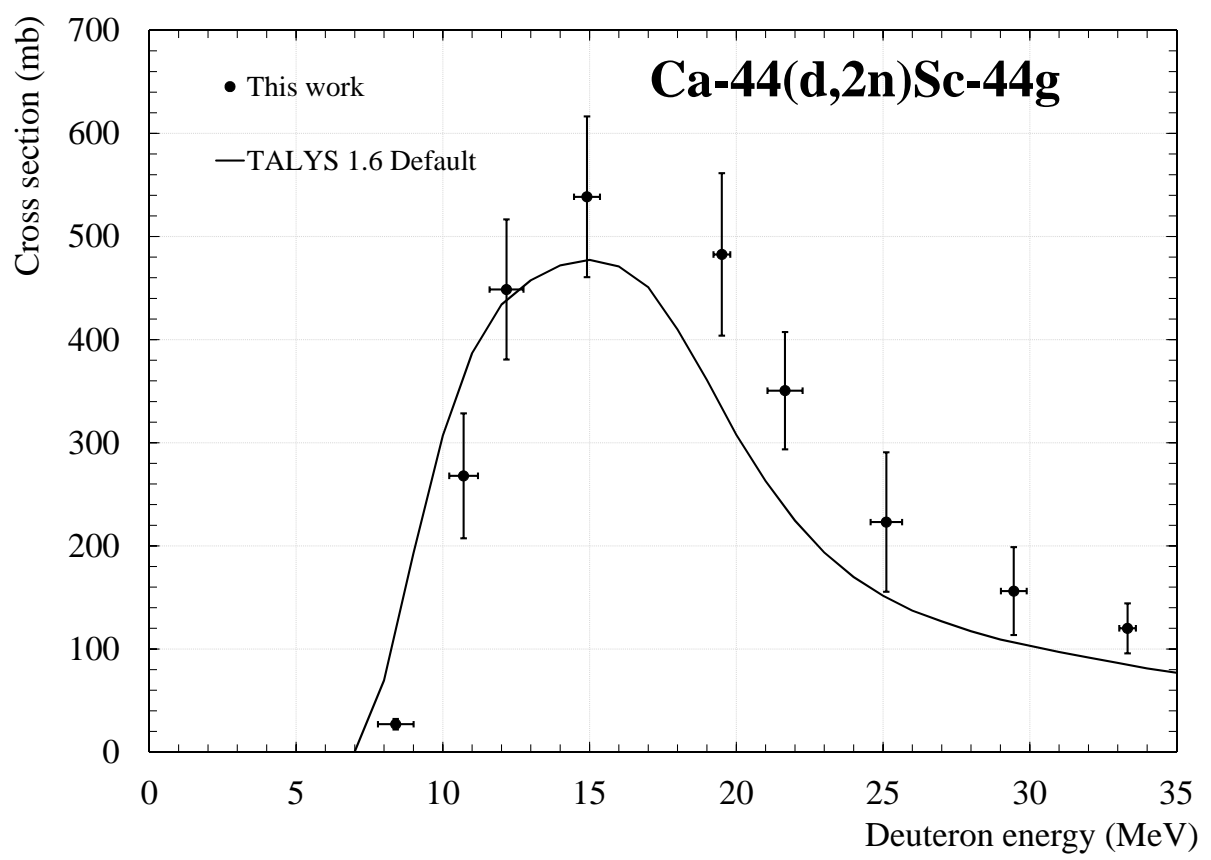

Figure 4: Excitation function for the Ca-44(d,2n)Sc-44g reaction (direct production)

\subsubsection{Production cross section of $K-4^{2}$}

K-42, with a half-life of 12.260 (3) hours, decays by $\beta$ - process $(100 \%)$ to Ca-42 (stable). It emits one main $\gamma$ ray at $1524.7 \mathrm{keV}$ with a branching ratio of $18 \%$. This gamma line has been used to determine the K-42 activity and deduce its production cross section, plotted in figure 5. The K-42 activity has not been obtained for all energies as it was not the main objective of this study. The TALYS code is not able to reproduce the excitation function for this reaction.

\subsubsection{Production cross section of $K-43$}

K-43 has a half-life of 22.3 (1) hours and decays by $\beta$ - emission at $100 \%$ to Ca-43 (stable), emitting four gamma rays with a branching ratio higher than $11 \%$ (see Table 3). Among these gammas, one is common to Sc-43. The three others gamma lines have been used to extract the cross section values. They also have permitted to deduce the K-43 contribution in the gamma line $(372.9 \mathrm{KeV})$, which is common with Sc-43. In figure 6, we can see that our experimental data are in agreement with the reaction energy threshold at $15.07 \mathrm{MeV}$. The TALYS code is not able to reproduce the experimental 


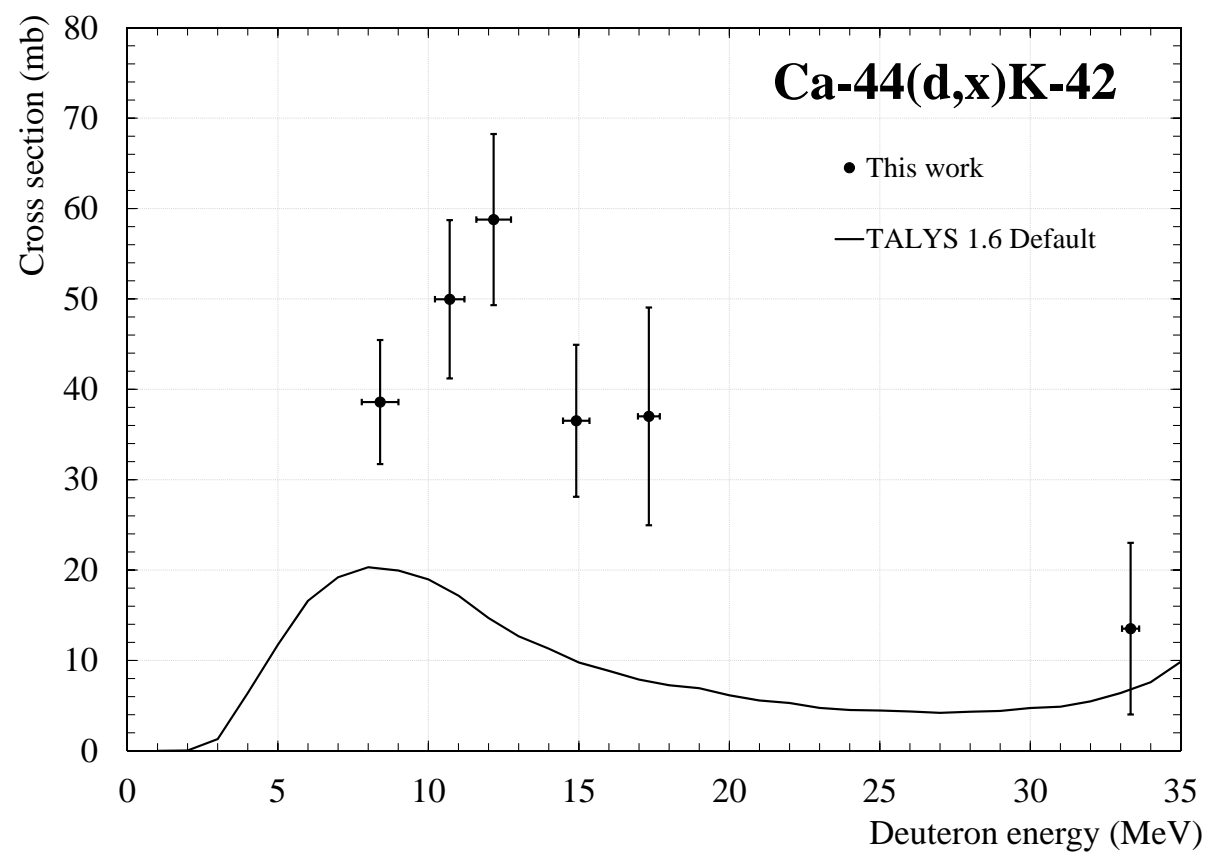

Figure 5: Excitation function for the Ca-44(d,x)K-42 reaction

trend and amplitude for this reaction.

\subsubsection{Production cross section of $S c-43$}

The Sc-43 has a half-life of $\mathrm{T}_{1 / 2}=3.891(12) \mathrm{h}$. It is a $88 \% \beta+$ emitter and decays to Ca-43 (stable), by emitting a $\gamma$ ray at $372.9 \mathrm{keV}$ (see Table 3 ) with a branching ratio of $22.5 \%$. This gamma line is common with K-43 which is also produced in the target (see part 3.1.4).

The activity of Sc-43 has been deduced from this peak using the other detectable gammas emitted by K-43, resulting on an additional uncertainty source for the determination of Sc-43 activity. As there is some cooling time of 14 hours between the end of irradiation and the activity measurements, the short half-life of Sc-43 and its low production cross section, don't permit to detect Sc-43 in every irradiated target. When the activity was high enough to be detected, the uncertainty on the peak fit was up to $30 \%$. Only two cross section values at 21.66 and $33.33 \mathrm{MeV}$ were measured and are reported in Table 4 . These values are plotted in figure 7 with the results of the TALYS code with default parameters. As no experimental data are available around the maximum plotted by TALYS, the amplitude of the curve can not be ver- 


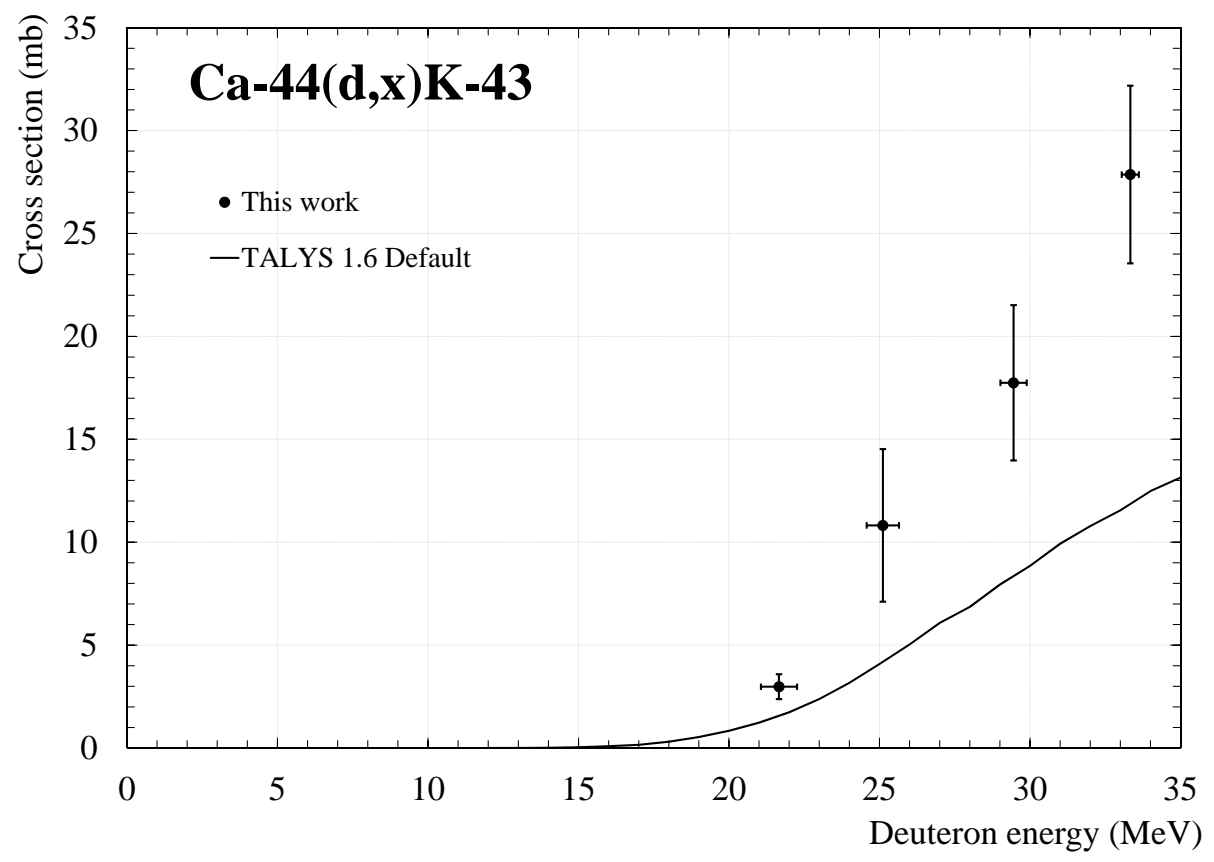

Figure 6: Excitation function for the Ca-44(d,x)K-43 reaction

ified. However, our existing experimental data points are in good agreement compared to the TALYS values. In addition, the Sc-43 is produced by a $(\mathrm{d}, \mathrm{xn})$ as well as Sc-44 for which the TALYS code shows a good reproduction of the trend.

\subsection{Thick Target production Yield of Sc-44}

Sc-44 can be produced using $\mathrm{Ca}-44$ with protons or deuterons as projectiles. The Thick Target production Yields of Sc-44g and Sc-44m using deuterons have been obtained from the experimental production cross sections determined in this work. They are plotted in figures 8 (a) and (b), respectively. These data are compared with the proton route, calculated from results summarized in databases (National Nuclear Data Center).

Using protons as projectiles at $30 \mathrm{MeV}$, the Sc-44g TTY calculated from the experimental cross section values of Mitchell et al., 1982, Levkovskij, 1991 and Krajewski et al., 2013, is $2.7 \mathrm{GBq} / \mu \mathrm{A} . \mathrm{h}$ (see figure 8 (a)). In this work, we calculate from our new cross section values obtained with deuterons a TTY of $2.9 \mathrm{GBq} / \mu \mathrm{A}$.h at $30 \mathrm{MeV}$, which is close to the value calculated in the case of protons. The cross section values obtained with the TALYS 


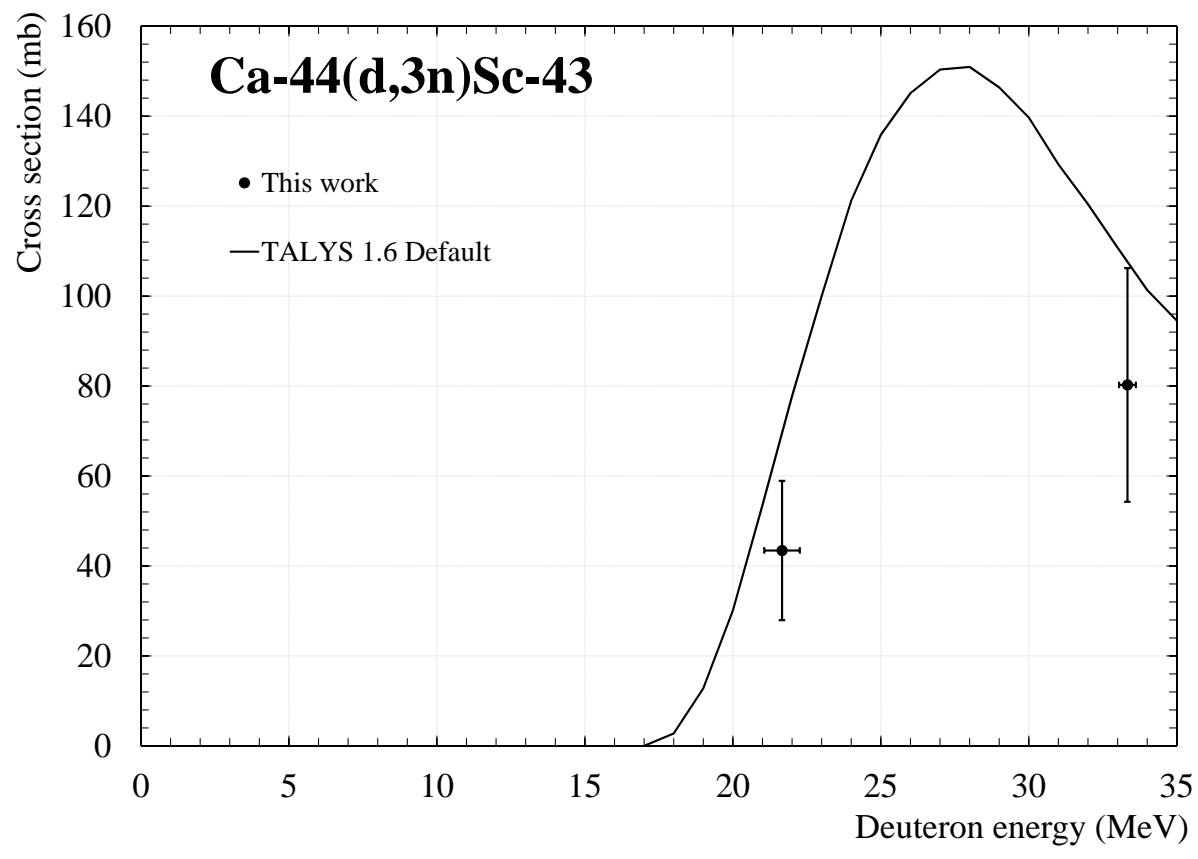

Figure 7: Excitation function for the Ca-44(d,3n)Sc-43 reaction

code lead to a TTY of $2.3 \mathrm{GBq} / \mu$ A.h (figure 2 (a)), i.e. $21 \%$ lower than the experimental value.

For the production of $\mathrm{Sc}-44 \mathrm{~m}$, the TTY is $23 \mathrm{MBq} / \mu \mathrm{A}$.h with protons at $30 \mathrm{MeV}$ (see figure 8 (b)). From our data using deuterons, the TTY is 70 $\mathrm{MBq} / \mu \mathrm{A}$.h with the same incident energy i.e. 3 times higher than the one obtained with protons. With the TALYS code results, the TTY is calculated to be $65 \mathrm{MBq} / \mu \mathrm{A} . \mathrm{h}, 7 \%$ lower than the experimental one.

These results show that higher Sc-44g production rate is obtained using protons instead of deuterons up to $27 \mathrm{MeV}$. With $30 \mathrm{MeV}$ protons and deuterons, similar amount of Sc-44g is produced with 2.7 and $2.9 \mathrm{MBq} / \mu \mathrm{A} . \mathrm{h}$, respectively. It must be taken into account that a smallest amount of carbonate calcium material is necessary with deuterons at $30 \mathrm{MeV}$ as compared to protons with the same energy. Indeed, the target thickness has to be 2.3 $\mathrm{mm}$ using deuterons against $4.1 \mathrm{~mm}$ using protons. In addition, the production of Sc-44m can significantly be increased using deuterons as projectiles instead of protons. 


\subsection{Sc-44 production scenarios}

Three production scenarios can be set and are presented below, depending of the use of Sc-44 and the cyclotron characteristics of commercially available machine (15 MeV, 30/15 MeV and 70/35 MeV proton/deuteron).

\subsubsection{Production of $S c-44 g$ for conventional PET imaging with small vector molecule}

The first scenario is the production of scandium- $44 \mathrm{~g}$ for conventional PET imaging for which Sc-44m has to be limited. Sc-43 is also of interest for PET and emits positrons with energies close to those of Sc-44g. Avoiding the production of Sc-43 is not an issue for this production scenario. The figure 8 (b) shows that the production of Sc- $44 \mathrm{~m}$ with protons is lower than with deuterons on the whole investigated energy range. This way, the use of protons is the best choice, compared with deuterons, to produce Sc-44g for PET imaging.

\subsubsection{Production of $S c-44 \mathrm{~g}$ for the new $3 \gamma$ imaging technique}

The second scenario is the production of scandium- $44 \mathrm{~g}$ for the new 3 gamma imaging technique using peptide or small molecule with rapid distribution on the body. In this case, Sc-44g is the radionuclide to produce in high quantity. The Sc-44m and Sc-43 production are not necessary as they don't have the third high energy $\gamma$ required for this new diagnosis technique. They will add noise on the detection system and an additional unnecessary dose to the patient. Sc- $44 \mathrm{~m}$ and Sc-43 can not be separated from Sc-44g in the target, contrary to the potassium isotopes (see figure 5 and figure 6).

With a $15 \mathrm{MeV}$ proton cyclotron. As shown in figure 8 (a), $1.9 \mathrm{GBq} / \mu \mathrm{A} . \mathrm{h}$ of Sc-44g is directly produced with $15 \mathrm{MeV}$ protons. The figure 9 shows the Sc-43 TTY calculated for protons as projectiles using the values published by Levkovskiy, 1991, and for deuterons using the TALYS 1.6 Default values plotted in figure 7 . Irradiating the target with a $15 \mathrm{MeV}$ proton beam leads to a Sc-43 production yield of $2.4 \mathrm{MBq} / \mu \mathrm{A} . \mathrm{h}$ and $10.5 \mathrm{MBq} / \mu \mathrm{A}$.h for Sc- $44 \mathrm{~m}$ (see figure 8 (b)). For these conditions of irradiation, the Sc-43 and Sc-44m production yields at the end of irradiation represent, respectively, $0.1 \%$ and $0.6 \%$ of the Sc-44g activity directly produced.

With a $30 \mathrm{MeV}$ proton - $15 \mathrm{MeV}$ deuteron cyclotron. With $15 \mathrm{MeV}$ deuterons, the amount of Sc-44g produced in the calcium-44 carbonate target is 680 
$\mathrm{MBq} / \mu \mathrm{A} . \mathrm{h}$ (see figure 8 (a)). For this energy, no Sc-43 can be produced as the Ca-44(d,3n)Sc-43 reaction threshold is $17.1 \mathrm{MeV} .11 \mathrm{MBq} / \mu \mathrm{A} . \mathrm{h}$ of Sc$44 \mathrm{~m}$ will be produced (see figure 8 (b)) which is $1.6 \%$ of the Sc- $44 \mathrm{~g}$ activity. With $30 \mathrm{MeV}$ protons, $2.7 \mathrm{GBq} / \mu \mathrm{A}$.h of Sc-44g is produced. The production yield of Sc-43 is $1.2 \mathrm{GBq} / \mu \mathrm{A} . \mathrm{h}$ (see figure 9) and $23 \mathrm{MBq} / \mu \mathrm{A} . \mathrm{h}$ for Sc-44m, which represent respectively $44 \%$ and $0.9 \%$ of the Sc- 44 g activity.

With a $30 \mathrm{MeV}$ deuteron cyclotron. With $30 \mathrm{MeV}$ deuteron, the Sc-44g TTY is $2.9 \mathrm{GBq} / \mu \mathrm{A} . \mathrm{h}$, close to that obtained with $30 \mathrm{MeV}$ protons. The amount of Sc-44m and Sc-43 produced are, respectively, $70 \mathrm{MBq} / \mu \mathrm{A} . \mathrm{h}$ and $600 \mathrm{MBq} / \mu \mathrm{A}$.h. This corresponds to 2.4 and $20.7 \%$ of Sc-44g activity.

\subsubsection{Production of the $S c-44 m / S c-44 g$ in-vivo generator for antibody la- belling}

The last scenario is about the production of the in-vivo generator Sc$44 \mathrm{~m} / \mathrm{Sc}-44 \mathrm{~g}$ for antibody labelling. A study has shown that the Sc-44m/Sc$44 \mathrm{~g}$ generator labelled with a DOTA-peptide is chemically stable and that Sc-44g stays attached to the chelator after the decay of its parent radionuclide Sc-44m (Huclier-Markai et al. in 2014). The in-vivo generator permits to extend the Sc-44 lifetime in the patient body. Sc-44m can then be used for antibody labelling for both conventional PET and $3 \gamma$ imaging. For this purpose, Sc-43 and Sc-44g direct production are useless since the transit time of antibodies is long as compared to their half-live.

The figure 10 shows that the $\mathrm{Sc}-44 \mathrm{~m} / \mathrm{Sc}-44 \mathrm{~g}$ cross section ratio (a) and the Sc-44m/Sc-44g TTY ratio(b) are higher using deuterons instead of protons.

In the specific case where an in-vivo generator Sc- $44 \mathrm{~m} / \mathrm{Sc}-44 \mathrm{~g}$ is used, a deuteron beam has to be prefered.

From a $15 \mathrm{MeV}$ deuteron beam, no Sc-43 can be produced and 680 $\mathrm{MBq} / \mu \mathrm{A} . \mathrm{h}$ of $\mathrm{Sc}-44 \mathrm{~g}$ is produced. The Sc-44m TTY for this energy is 11 $\mathrm{MBq} / \mu \mathrm{A} . \mathrm{h}$. It is 62 times less than the $\mathrm{Sc}-44 \mathrm{~g}$ activity.

With $30 \mathrm{MeV}$ deuteron beam, $70 \mathrm{MBq} / \mu \mathrm{A}$.h of $\mathrm{Sc}-44 \mathrm{~m}$ is produced. The figure 11 shows the decay of Sc-44m, Sc-44g and Sc-43 taking into account the initial activity produced with a $30 \mathrm{MeV}$ deuteron beam. The secular equilibrium between Sc-44m and Sc-44g is reached after 50 hours, which is close to one Sc-44m half-life, i.e. 2.44 days. Waiting for this time after the end of irradiation, the Sc-43 production decreases to $18 \mathrm{kBq} / \mu \mathrm{A} . \mathrm{h}(0.05 \%$ of Sc- $44 \mathrm{~m}$ activity) and the Sc-44g direct production decreases to $2.3 \mathrm{kBq} / \mu \mathrm{A} . \mathrm{h}$. 
The Sc-44m production, waiting for 2.44 days after the end of irradiation reaches $35 \mathrm{MBq} / \mu \mathrm{A}$.h, which is 3.2 times higher than the one obtained with $15 \mathrm{MeV}$ deuterons at the time of the end of irradiation.

However, only few accelerators in the world are able to deliver $30 \mathrm{MeV}$ deuterons, but the technology is available. This type of cyclotron permits to produce a higher amount of Sc-44m than using $15 \mathrm{MeV}$ deuterons or a proton beam.

\section{Conclusion}

New experimental cross section values have been presented for the Ca$44(\mathrm{~d}, \mathrm{x})$ Sc- $44 \mathrm{~m}, \mathrm{Sc}-44 \mathrm{~g}$ reactions with some informations on the Sc-43, K-42 and K-43 contaminants. The TALYS results are close to experimental values for the Ca-44(p,n) and Ca-44(d,2n) reactions. New measurements dedicated to Sc-43 with deuterons as projectiles are needed to obtain experimental values on this contaminant production cross section, and validate, or not, the trend made by the TALYS code. However, TALYS with default models is not able to reproduce the data for the production of potassium isotopes.

These new sets of experimental data allow to calculate Thick Target production Yields and compare the proton and deuteron routes for three different Sc-44 production scenarios. This article shows that the use of proton beam is the best choice, as compared to deuterons, to produce Sc-44g for PET imaging using peptide or small molecules with rapid distribution in the body. For the new $3 \gamma$ imaging technique developed at the SUBATECH laboratory, Sc$44 \mathrm{~g}$ has to be produce with protons of $15 \mathrm{MeV}$ to limit background generated by the Sc-44m and Sc-43 decay.

The production of the Sc-44m/Sc-44g in-vivo generator for antibody labelling required the highest Sc- $44 \mathrm{~m}$ production rate, with a limited amount of Sc-44g directly produced. Our new experimental results have shown that the Sc- $44 \mathrm{~m} / \mathrm{Sc}-44 \mathrm{~g}$ cross section and TTY ratios are higher with deuterons than with protons, whatever the incident beam energy. The production of $\mathrm{Sc}-44 \mathrm{~m}$ for the in-vivo generator is advantageous with deuterons as projectiles, using a calcium- 44 carbonate target. Sc- $44 \mathrm{~m}$ can be produced with $15 \mathrm{MeV}$ deuterons beam but a higher amount of $\mathrm{Sc}-44 \mathrm{~m}$ is produced with a $30 \mathrm{MeV}$ deuteron beam. With this last energy value, some cooling time before the extraction and separation processes permits to better reduce the contribution of directly produced Sc- $44 \mathrm{~g}$ activity and obtain, by the way, a negligible level of Sc-43. 


\section{Acknowledgments}

The ARRONAX cyclotron is a project promoted by the Regional Council of Pays de la Loire financed by local authorities, the French government and the European Union. This work has been, in part, supported by a grant from the French National Agency for Research called "Investissements d'Avenir", Equipex Arronax-Plus no ANR-11-EQPX-0004 and Labex $\mathrm{n}^{o}$ ANR-11-LABX-0018-01. Authors would like to thanks the Institut de Physique Nucléaire d'Orsay (IPNO) and the CACAO (Chimie des Actinides et Cibles radioActives à Orsay) project team for the carbonate calcium target manufacturing. 

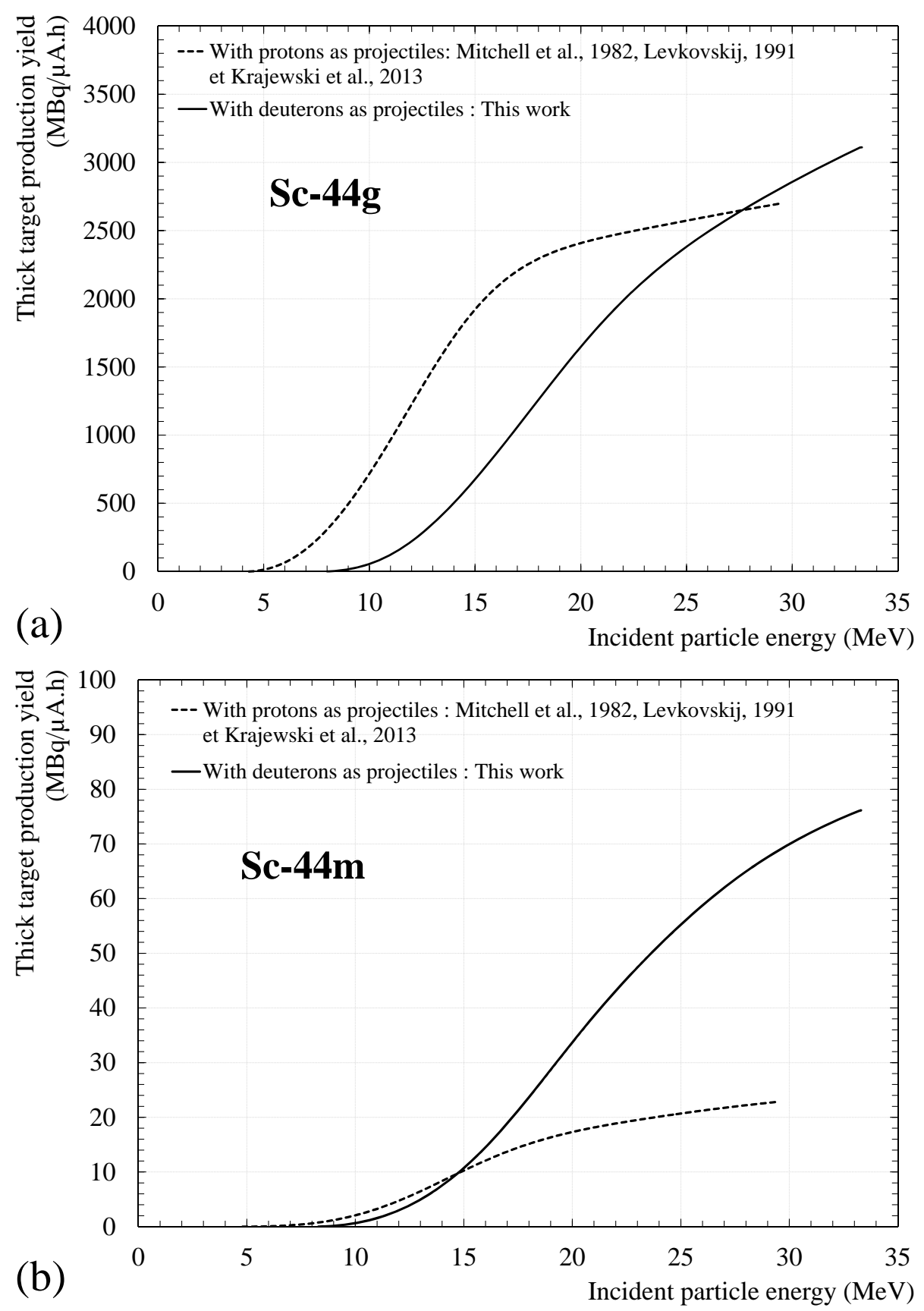

Figure 8: Thick Target production Yields for the Ca-44(d,2n)Sc-44g (a) and Ca44(d,2n)Sc-44m (b) reactions, calculated with the cross section values of this work. Comparison with the Ca-44(p,n)Sc-44g (a) and Ca-44(p,n)Sc-44m (b) reactions. 


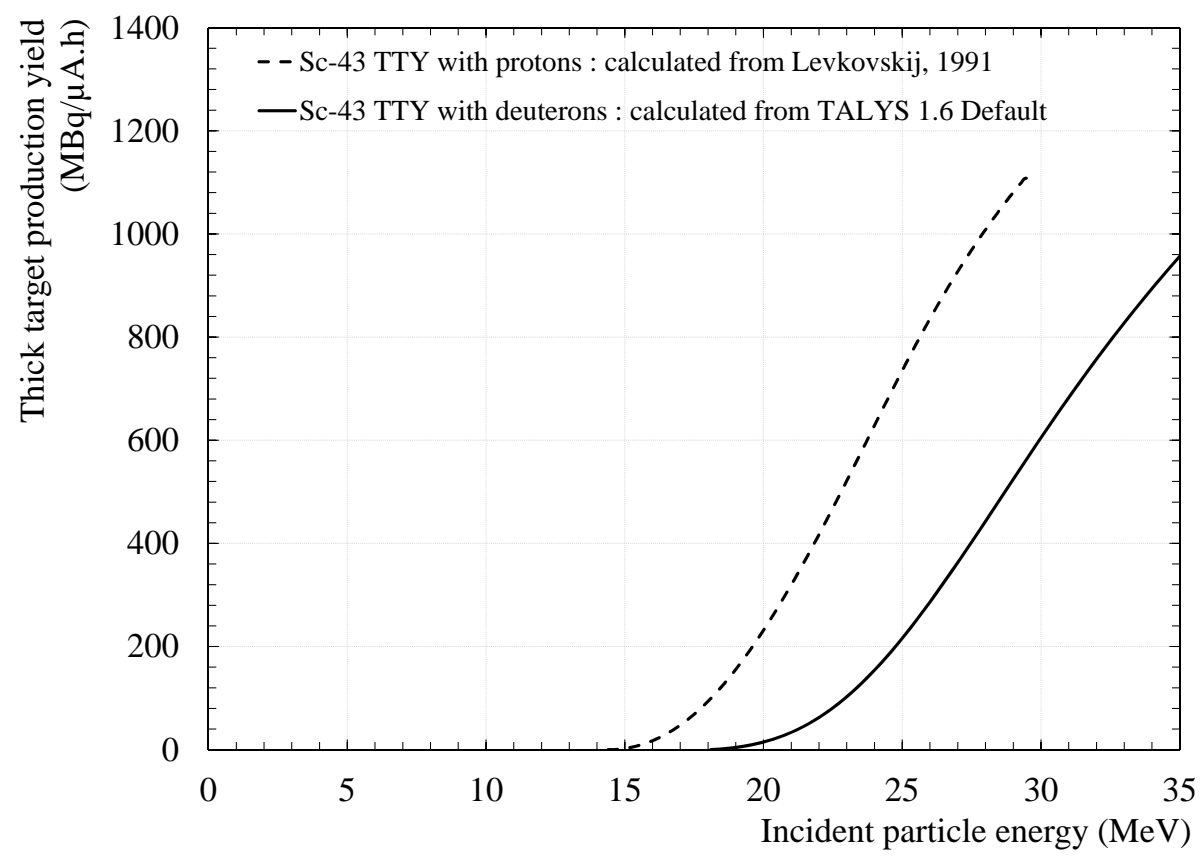

Figure 9: Thick Target production Yields for the Ca-44(d,3n)Sc-43 and the Ca-44(d,2n)Sc43 reactions, calculated with the cross section values of levkovskij, 1991 for the proton route and with the TALYS 1.6 Default results for the deuteron route 

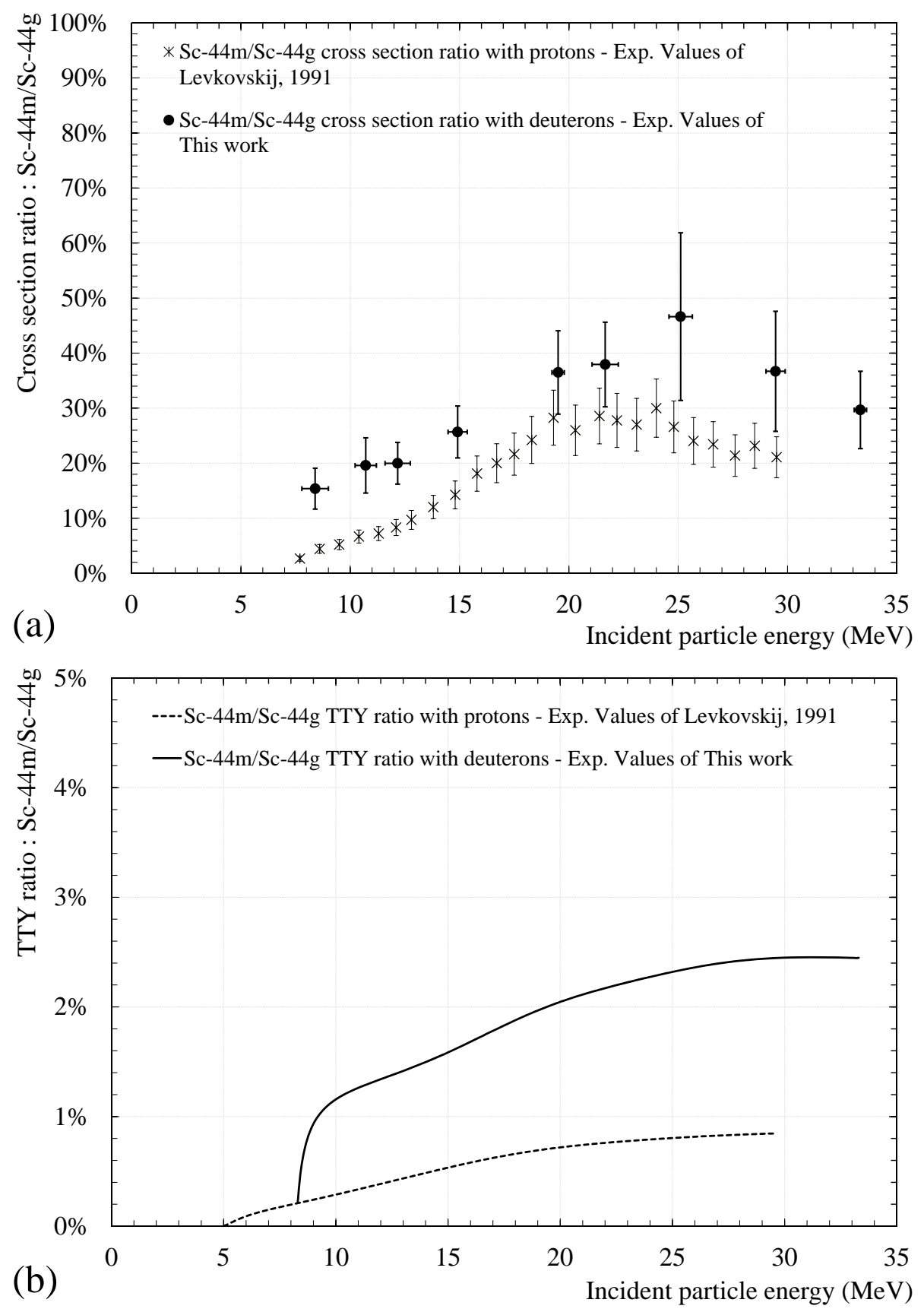

Figure 10: Experimental Sc-44m/Sc-44g cross section (a) and calculated TTY (b) ratios using protons and deuterons as projectiles 


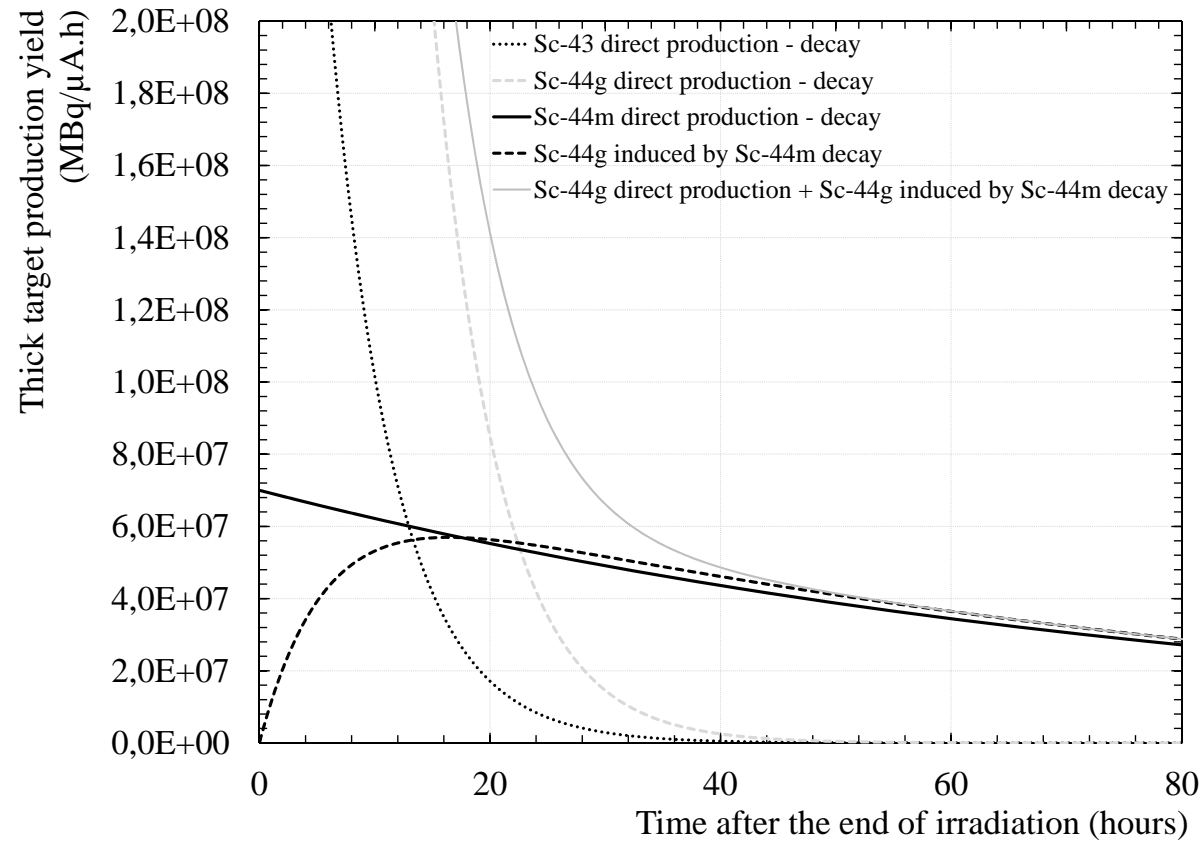

Figure 11: Sc-44m, Sc-44g and Sc-43 decay from an initial activity produced with a 30 $\mathrm{MeV}$ deuteron beam 

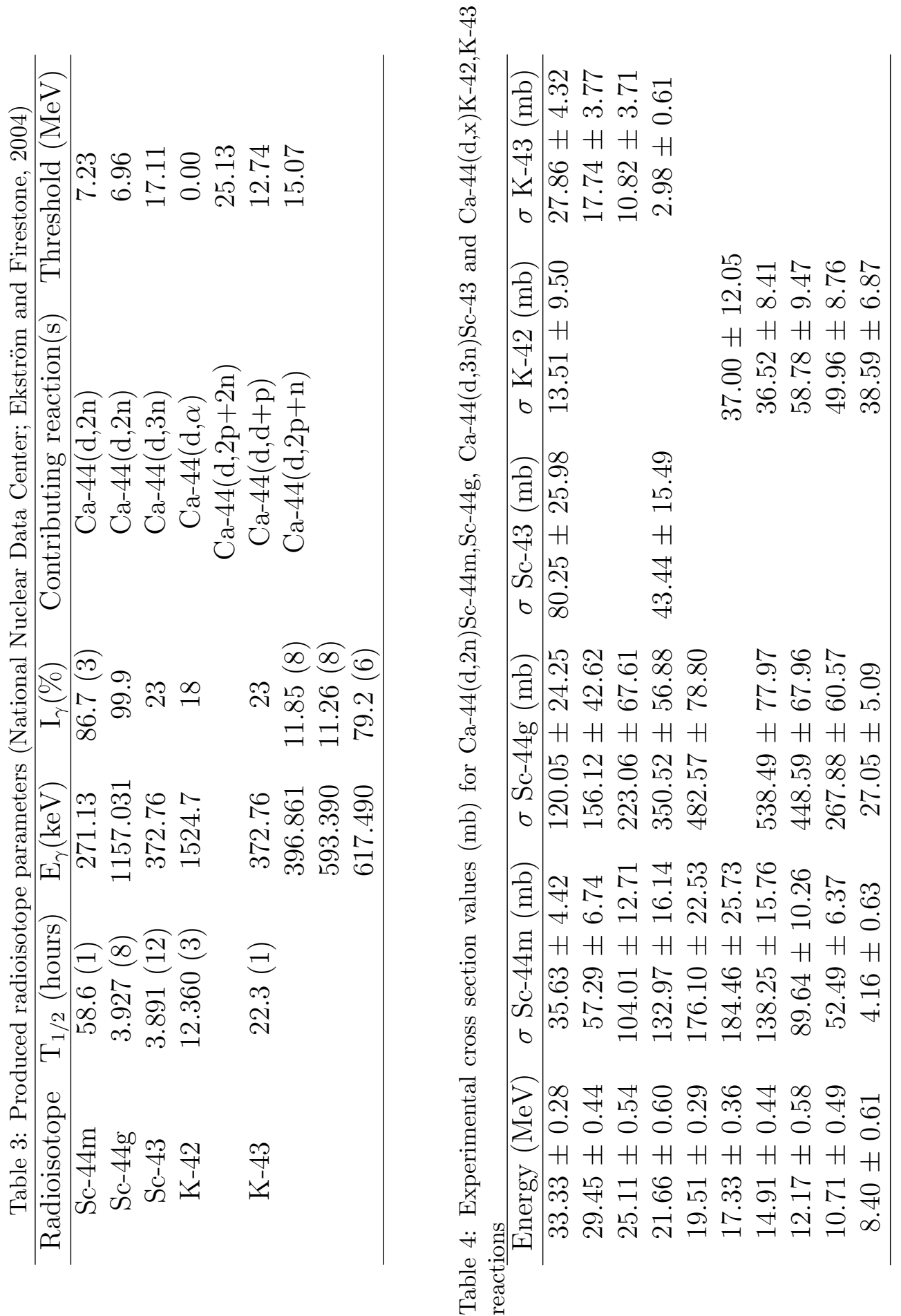


\section{References}

Alliot, C., Audouin, N., Barbet, J., Bonraisin, A.C., Bossé, V., Bourdeau, C., Bourgeois, M., Duchemin, C., Guertin, A., Haddad, F., Huclier-Markai, H., Kerdjoudj, R., Laizé, J., Métivier, V., Michel, N. and Mokili, M., 2015. Is there an interest to use deuteron beams to produce non-conventional radionuclides?, Frontiers in Medicine doi: 10.3389/fmed.2015.00031.

Blessing, G., Bräutigam, W., Böge, H.G., Gad, N., Scholten, B., and Qaim, S.M., 1995. Internal irradiation system for excitation function measurement via the stacked-foil technique, Appl. Radiat. Isot. 955, 46-9.

Chemgas@, major supplier of enriched stable isotopes: http://www.chemgas.com/

Duchemin C., Guertin A., Haddad F., Michel N. and Métivier V., 2015. Cross section measurements of deuteron induced nuclear reactions on natural tungsten up to $34 \mathrm{MeV}$, Applied Radiation and Isotopes 97 (2015) 52-58

Duchemin C., Guertin A., Haddad F., Michel N. and Métivier V., 2015. Production of medical isotopes from a thorium target irradiated by light charged particles up to $70 \mathrm{MeV}$, Phys. Med. Biol. 60, 931-946.

Durnez A., Petitbon-Thévenet V. and Fortuna F., 2014. Preparation of molybdenum target by centrifugal method, J. Radioanal. Nucl. Chem. 299:1149-1154.

Ekström, L.F., and Firestone, R.B., 2004. Information extracted from the Table of Radioactive Isotopes, version 2.1.

FitzPeaks Gamma Analysis and Calibration Software version 3.66, produced by JF Computing Services (UK), based on methods presented in Nucl.Instrum. and Methods (1981) 190, 89-99, describing the program SAMPO80 of the Helsinki University of Technology, Finland.

Gallego Manzano, L., Bassetto, S., Beaupere, N., Briend, P., Carlier, T., Chérel, M., Cussonneau, J-P., Donnard, J., Gorski, M., Hamanishi, R., Kraeber-Bodéré, F., LeRaya, P., Lemaire, O., Masbou, J., Mihara, S., Morteau, E., Scotto Lavina, L., Stutzmann, J-S., Tauchi, T., Thers, D., 2014. XEMIS: A liquid xenon detector for medical imaging, Nuclear Instruments and Methods in Physics Research A, doi:10.1016/j.nima.2014.11.040.

Grignon C., Barbet J., Bardies M., et al. (2007). Nuclear medical imaging using beta + gamma coincidences from Sc-44 radio-nuclide with liquid xenon as detector medium, Nucl. Instrum. Meth. A 571, 142-145.

Haddad, F., Ferrer, L., Guertin, A., Carlier, T., Michel, N., Barbet, J., Chatal, J.F., 2008. Arronax a high-energy and high-intensity cyclotron for nuclear medicine, Eur. J. Nucl. Med. Mol. Imaging 35, 1377-1387. 
Huclier-Markai S., Kerdjoudj R., Alliot C., Bonraisin A.C., Michel N., Haddad F., Barbet J., 2014. Optimization of reaction conditions for the radiolabelling of DOTA and DOTA-peptide with $44 \mathrm{~m} / 44 \mathrm{Sc}$ and experimental evidence of the feasibility of an in vivo PET generator. Nuclear Medicine and Biology 41, e36-e43.

Koning, A.J., Rochman, D., 2012. Modern nuclear data evaluation with the TALYS code system, Nucl. Data Sheets 113, 2841.

Krajewski, S., Cydzik, I., Abbas, K., Bulgheroni, A., Simonelli, F., Holzwarth, U., Bilewicz, A., 2013. Cyclotron production of 44Sc for clinical application, Radiochimica Acta, Vol.101, p.333.

Levkovskij, V.N, 1991. Activation cross section nuclides of average masses $(\mathrm{A}=40-100)$ by protons and alpha-particles with average energies $(\mathrm{E}=10-50$ $\mathrm{MeV}$ )., Reference Book: Levkovskij,Act.Cs.By Protons and Alphas,Moscow 1991.

Majkowska-Pilip A. and Bilewicz A., 2011. Macrocyclic complexes of scandium radionuclides as precursors for diagnostic and therapeutic radiopharmaceuticals. J. Inorg. Biochem., 105.

Mitchell L.W., Anderson M.R., Kennett S.R., and Sargood D.G, 1982. Cross sections and thermonuclear reaction rates for $42 \mathrm{Ca}(\mathrm{p}) ,43 \mathrm{Ca}, 44 \mathrm{Ca}(\mathrm{p}$, 45Sc, 44Ca(p,n) 44Sc and 45Sc(p,n)45Ti. Nuclear Physics A, 380.

Müller C., Bunka M., Haller S., Köster U., Groehn V., Bernhardt P., Van der Meulen N., Türler A., and Schibli R., 2014. Promising prospects for $44 \mathrm{Sc}-/ 47 \mathrm{Sc}-$ based theragnostics : Application of $47 \mathrm{Sc}$ for radionuclide tumor therapy in mice. J Nucl Med, 55.

National Nuclear Data Center, information extracted from the Experimental Nuclear Reaction Data (EXFOR) Database, http://www.nndc.bnl.gov/exfor/exfor.htm, Version of November 05, 2013.

Oger T. et al., 2012. A liquid xenon tpc for a medical imaging compton telescope, Nucl.Instrum.Meth. A, 695.

Petitbon-Thévenet V., 2010. The target manufacturing laboratory at the IPNO, Target and Stripper Foil Technologies for High Intensity Beams - Proceedings of the 24th World Conference of the International Nuclear Target Development Society, Nuclear Instruments and Methods in Physics Research Section A: Accelerators, Spectrometers, Detectors and Associated Equipment, Volume 613, Issue 3, Pages 419-422.

Roesch F. et al., 2012. Scandium-44: benefits of a long-lived PET radionuclide available from the (44)Ti/(44)Sc generator system., Curr Radiopharm., 5(3):187-201. 
Tárkányi, F., Takács, S., Gul, K., Hermanne, A., Mustafa, M.G., Nortier, M., Oblozinsky, P., Qaim, S.M., Scholten, B., Shubin, Yu.N., Youxiang, Z., 2001. Beam monitor reactions, in Charged Particle Cross Section Database for Medical Radioisotope Production: Diagnostic Radioisotopes and Monitor Reactions; IAEA-TECDOC-1211, pages 49-152, IAEA, Vienna. Database available on https://www-nds.iaea.org/medportal/, update may 2013.

Tárkányi, F., Sonck, M. and Hermanne, A., 2002. Investigation of the natMo $(\mathrm{p}, \mathrm{x}) 96 \mathrm{mgTc}$ nuclear reaction to monitor proton beams: New measurements and consequences on the earlier reported data, Nuclear Instruments and Methods B 198, 183.

Ziegler, J.F., Ziegler, M.D. and Biersack, J.P., 2010. SRIM The stopping and range of ions in matter, Nucl. Instrum. Methods Phys. Res., Sect. B $268,1818-1823$. 